\title{
AN INTEGRAL-EQUATION APPROACH FOR DEFAULTABLE BOND PRICES WITH APPLICATION TO CREDIT SPREADS
}

\author{
YU-TING CHEN, ${ }^{*}$ National Chiao Tung University \\ CHENG-FEW LEE, ${ }^{* *}$ Rutgers University and National Chiao Tung University \\ YUAN-CHUNG SHEU, ${ }^{* * *}$ National Chiao Tung University
}

\begin{abstract}
We study defaultable bond prices in the Black-Cox model with jumps in the asset value. The jump-size distribution is arbitrary, and following Longstaff and Schwartz (1995) and Zhou (2001) we assume that, if default occurs, the recovery at maturity depends on the 'severity of default'. Under this general setting, the vehicle for our analysis is an integral equation. With the aid of this, we prove some properties of the bond price which are consistent numerically and empirically with earlier works. In particular, the limiting credit spread as time to maturity tends to 0 is nonzero. As a byproduct, we show that the integral equation implies an infinite-series expansion for the bond price.
\end{abstract}

Keywords: Jump diffusion; default barrier; bond price; credit spread

2000 Mathematics Subject Classification: Primary 60J25; 60J75; 60G44

\section{Introduction}

Our goal is to study the price of a defaultable bond issued by a given firm. For simplicity, we assume that the bond is a zero-coupon bond with one dollar face value and time to maturity $T$. First we consider an extension of the Merton model due to Black and Cox [3]. The asset value process $V$ of the firm, under the risk-neutral measure, follows the dynamic

$$
\mathrm{d} V_{t}=(r-a) V_{t} \mathrm{~d} t+\sigma V_{t} \mathrm{~d} W_{t} .
$$

Here, $a$ is the payout rate of the firm, $r>0$ is the risk-free interest rate, the square of $\sigma>0$ is the instantaneous volatility of the return on the firm's asset, and $W$ is a standard Brownian motion. To allow the possibility that default may occur at any time before maturity, Black and Cox assumed the existence of a default boundary:

$$
K_{t}=K_{0} \mathrm{e}^{\kappa t}, \quad 0 \leq t \leq T,
$$

where $\kappa \in \mathbb{R}$. (We note that Longstaff and Schwartz [8] considered a constant boundary.) The default time is then defined by

$$
\tau(T)=\inf \left\{0 \leq t \leq T ; V_{t} \leq K_{t}\right\}
$$

Received 24 August 2007; revision received 17 November 2008.

* Current address: Institute of Mathematics, Academia Sinica, Nankang, Taipei, Taiwan.

** Postal address: Department of Finance, Rutgers University, New Brunswick, NJ, USA.

*** Postal address: Department of Applied Mathematics, National Chiao Tung University, Hsinchu, Taiwan.

Email address: sheu@math.nctu.edu.tw 
with the convention that inf $\varnothing=+\infty$. The recovery at maturity is $\min \left(V_{T}, 1\right)$ if there is no default up to time $T$. However, if there is default, that is, the boundary is hit before or at the maturity, bond holders take over the firm and own the remaining value of the firm, that is, $K_{\tau(T)}$. The bond price under this setting can be easily calculated because of the analytic tractability of Brownian motions. For details, see [6, Section 2.6].

To take into account empirical phenomena such as the asymmetric leptokurtic feature of the distribution of asset returns, we assume throughout this paper that, under a given risk-neutral measure $\mathrm{P}$, the value $V$ of the firm follows the dynamic

$$
\mathrm{d} V_{t}=V_{t-}\left[r \mathrm{~d} t+\sigma \mathrm{d} W_{t}+\mathrm{d}\left(\sum_{i=1}^{N_{t}} U_{i}-\lambda \nu t\right)\right],
$$

where $N=\left(N_{t}\right)$ is a Poisson process with intensity $\lambda$ and $\left\{U_{i}\right\}$ is a sequence of independent random variables with distribution supported on $(-1,0) \cup(0, \infty)$ which has mean $v$. The three elements $W, N$, and $\left\{U_{i}\right\}$ are independent. If there is default, as in [8] and [11], we assume that the payoff is offset by a function of the 'severity of default'. Furthermore, for simplicity, we assume, as in [11], that the payment is always made at the time to maturity. In other words, the payoff of the zero-coupon bond at time $T$ is given by

$$
\mathbf{1}(\tau(T)>T)+\left[1-\psi\left(\log \left(\frac{V_{\tau(T)}}{K_{\tau(T)}}\right)\right)\right] \mathbf{1}(\tau(T) \leq T) .
$$

(We can think of $\psi(\log x)$ as the sume of bankruptcy costs and others.) Set $X_{t}=\log \left(V_{t} / K_{t}\right)$. Then it follows from (1.4) that the no-arbitrage price of the bond is given by

$$
D\left(V_{0}, T\right)=\mathrm{e}^{-r T}-\mathrm{e}^{-r T} \mathrm{E}_{x}\left[\psi\left(X_{\tau(T)}\right) \mathbf{1}(\tau(T) \leq T)\right]=\mathrm{e}^{-r T}-\mathrm{e}^{-r T} \Phi(x, T),
$$

where $x=\log \left(V_{0} / K_{0}\right), \tau(t)=\inf \left\{0 \leq s \leq t ; X_{s} \leq 0\right\}$, and

$$
\Phi(y, t)=\mathrm{E}_{y}\left[\psi\left(X_{\tau(t)}\right) \mathbf{1}(\tau(t) \leq t)\right] .
$$

Here, $\mathrm{E}_{y}$ denotes the expectation conditioned on $X_{0}=y$ under the risk-neutral measure $\mathrm{P}$. The notation $\mathrm{P}_{y}$ is similarly defined. To study the bond price in (1.5), we will focus on the function $\Phi$ from now on.

In the literature, $1-\psi$ is termed as the recovery rate. It is worth noting that in empirical studies, even for the same class of bond issues, the realized recovery rate differs significantly over different time periods and different firms. See [1] and [4]. By taking $\psi$ to be a function of the form $a-b \mathrm{e}^{y}$, Zhou [11] provided a Monte Carlo simulation scheme to compute several characteristics of the bond price. Zhou [11] found within the results that, by manipulating the parameters, various shapes of credit spreads, default probabilities, and other properties formerly observed in empirical studies can be recovered. Among the significant results is the possibility to exhibit nonzero spread for small time to maturity. (Diffusion models for risky bond pricing have been criticized for years because of the failure to capture this phenomenon.) Besides the framework of the Black-Cox model, Hilberink and Rogers [5] considered the Leland model, which is a generalization of the Black-Cox model. See [7]. In this case, in addition to a recovery structure which is slightly different from the aforementioned one, there are coupons paid continuously up to the default time. In [5] the authors generalized the Leland model by assuming that the logarithm of the firm's asset value is an independent sum of a Brownian motion with drift and a downward-jump compound Poisson process. Although there is no closed-form 
solution for the bond price, they obtained its Fourier transform. They also observed in their numerical results that the limiting credit spread, as the time to maturity tends to 0 , is positive and gave a proof of this result.

In this paper, instead of seeking the closed-form solution for the bond price, we study several aspects of the bond with the help of an integral equation satisfied by the function $\Phi$ in (1.6). The elements of the integral equation have forms which allows further analysis. (See Theorem 2.2, below.) Using these elements, we study some analytic properties of the bond price. In particular, under mild conditions on the jump distribution and the function $\psi$, we show in Theorem 3.1 that the credit spread has a strictly positive lower bound as the time to maturity tends to 0 . It is interesting to compare our results with those obtained in [5] and [11], where in both articles only some specific jump distributions and recoveries were considered. As another application of the integral equation, we give an infinite-series expansion for the bond price. (Note that in Merton [9] where $1+U_{j}$ is lognormal, the price of the risky zero-coupon bond is an infinite series for which each summand takes the form of a Black-Scholes European option price. See Theorem 9.3.1 of [2].)

The paper is organized as follows. In Section 2 we derive an integral equation from a decomposition of the event that default occurs. Based on the results in Section 2, in Section 3 we study some analytic properties of the bond price and investigate the asymptotic behavior of its credit spread as the time to maturity tends to 0. In Appendix A we give an infinite-series expansion for the bond price.

\section{An integral equation for $\Phi$}

We begin with the unique (up to indistinguishability) solution to (1.3), which is given by

$$
V_{t}=V_{0} \exp \left\{\left(r-\frac{1}{2} \sigma^{2}-\lambda \nu\right) t+\sigma W_{t}\right\} \prod_{j=1}^{N_{t}}\left(1+U_{j}\right)
$$

Recall that $X=\log (V / K)$. For the diffusion part of $X$, we set $\mu=r-\frac{1}{2} \sigma^{2}-\lambda \nu-\kappa$ and $X_{t}^{c}=\mu t+\sigma W_{t}$ for all $t \in \mathbb{R}_{+}$. For the jump part of $X$, we set $Y_{n}=-\log \left(1+U_{n}\right)$ and $Z_{t}=\sum_{n=1}^{N_{t}} Y_{n}$. Then

$$
X_{t}=X_{0}+X_{t}^{c}-Z_{t} \quad \text { for all } t \in \mathbb{R}_{+},
$$

where $X_{0}=\log \left(V_{0} / K_{0}\right)$.

For any $x \in \mathbb{R}$, write $\hat{x}=x / \sigma$. Let $J_{k}$ denote the $k$ th epoch time of the compound Poisson process $Z$ with interarrival time $S_{k}$, that is, $J_{k}=\sum_{j=1}^{k} S_{j}$. Furthermore, if $G_{1}, \ldots, G_{n}$ are random variables on $\mathbb{R}$, we write $F_{G_{1}, \ldots, G_{n}}$ for their joint distribution. We preserve $F$ for the distribution of $Y_{1}$.

Now, write

$$
[\tau(T) \leq T]=A \cup B \cup C \cup D,
$$

where

$$
\begin{array}{ll}
A=\left[\tau(T) \leq T<J_{1}\right] & \text { (no jump up to maturity and default is caused by diffusion), } \\
B=\left[\tau(T)<J_{1} \leq T\right] & \text { (jump occurs up to maturity and default occurs before } \left.J_{1}\right), \\
C=\left[\tau(T)=J_{1} \leq T\right] & \text { (jump occurs up to maturity and default occurs at } \left.J_{1}\right), \\
D=\left[J_{1}<\tau(T) \leq T\right] & \text { (jump occurs up to maturity and default occurs after } \left.J_{1}\right) .
\end{array}
$$


Note that $\{A, B, C, D\}$ is a partition of $[\tau(T) \leq T]$. With these events defined, we set $G_{A}(x, T)=\mathrm{E}_{x}\left[\psi\left(X_{\tau(T)}\right) ; A\right]$, and similarly for $G_{B}, G_{C}$, and $G_{D}$. Before stating our results, we recall some facts about the joint distribution of the Brownian motion with drift and its maximum process for the convenience of readers. For details and proofs, see [10, pp. 113-115].

Theorem 2.1. Let $\alpha \in \mathbb{R}$ and $T>0$, let $W(t ; \alpha)=\alpha t+W(t)$, and let $M(T ; \alpha)=$ $\max _{0 \leq t \leq T} W(t ; \alpha)$. Then the joint density of $M(T ; \alpha)$ and $W(T ; \alpha)$ is given by

$$
\begin{aligned}
& f_{M(T ; \alpha), W(T ; \alpha)}(m, w) \\
& \quad= \begin{cases}\frac{2(2 m-w)}{T \sqrt{2 \pi T}} \exp \left\{\alpha w-\frac{1}{2} \alpha^{2} T-\frac{1}{2 T}(2 m-w)^{2}\right\}, & w \leq m, m \geq 0, \\
0, & \text { otherwise. }\end{cases}
\end{aligned}
$$

Therefore, the density of $M(T ; \alpha)$ is given by

$$
f_{M(T ; \alpha)}(m)= \begin{cases}\frac{2}{\sqrt{2 \pi T}} \exp \left\{-\frac{1}{2 T}(m-\alpha T)^{2}\right\}-2 \alpha \mathrm{e}^{2 \alpha m} \mathcal{N}\left(\frac{-m-\alpha T}{\sqrt{T}}\right), & m \geq 0, \\ 0, & \text { otherwise, }\end{cases}
$$

and

$$
\mathrm{P}[M(T ; \alpha) \leq m]= \begin{cases}\mathcal{N}\left(\frac{m-\alpha T}{\sqrt{T}}\right)-\mathrm{e}^{2 \alpha m} \mathcal{N}\left(\frac{-m-\alpha T}{\sqrt{T}}\right), & m \geq 0, \\ 0, & m<0 .\end{cases}
$$

Here $\mathcal{N}(\cdot)$ is the cumulative distribution function of the standard normal distribution.

Proposition 2.1. We have the following representations of $G_{A}, G_{B}$, and $G_{C}$ :

$$
\begin{aligned}
& G_{A}(x, T)=\psi(0) \mathrm{e}^{-\lambda T}\left(\mathcal{N}\left(\frac{-\hat{x}-\hat{\mu} T}{\sqrt{T}}\right)+\mathrm{e}^{-2 \hat{\mu} \hat{x}} \mathcal{N}\left(\frac{-\hat{x}+\hat{\mu} T}{\sqrt{T}}\right)\right) \\
& G_{B}(x, T)=\psi(0)\left(\int_{0}^{T} \mathcal{N}\left(\frac{-\hat{x}-\hat{\mu} t}{\sqrt{t}}\right) \mathrm{d} F_{J_{1}}(t)+\int_{0}^{T} \mathrm{e}^{-2 \hat{\mu} \hat{x}} \mathcal{N}\left(\frac{-\hat{x}+\hat{\mu} t}{\sqrt{t}}\right) \mathrm{d} F_{J_{1}}(t)\right) \\
& G_{C}(x, T)=\int_{0}^{T} \mathrm{~d} F_{J_{1}}(t) \int_{0}^{\infty} \mathrm{d} F(y) \int_{0}^{y} \mathrm{~d} w \psi(w-y) H(x, w, t)
\end{aligned}
$$

where

$$
H(x, w, t)=g\left(x-w+\mu t ; t \sigma^{2}\right)-\mathrm{e}^{-2 \hat{\mu} \hat{x}} g\left(x+w-\mu t ; t \sigma^{2}\right)
$$

and

$$
g\left(y ; \varrho^{2}\right)=\frac{1}{\sqrt{2 \pi \varrho}} \exp \left\{-\frac{y^{2}}{2 \varrho^{2}}\right\} .
$$

Proof. Note that $\psi\left(X_{\tau(T)}\right)=\psi(0)$ on $A$ and $B$. By the independence of $\left\{W_{t} ; t \in \mathbb{R}_{+}\right\}$and $J_{1}$, we obtain

$$
\begin{aligned}
G_{A}(x, T) & =\mathrm{P}\left[T<J_{1}\right] \psi(0) \mathrm{P}\left[\min _{s \leq T} x+\mu s+\sigma W_{s} \leq 0\right] \\
& =\mathrm{P}\left[T<J_{1}\right] \psi(0) \mathrm{P}\left[\max _{s \leq T}-x-\mu s-\sigma W_{s} \geq 0\right] \\
& =\mathrm{P}\left[T<J_{1}\right] \psi(0) \mathrm{P}\left[\max _{s \leq T}-\hat{\mu} s+W_{s} \geq \hat{x}\right],
\end{aligned}
$$


where the last equality follows from the symmetry of standard Brownian motion. By (2.5), we have

$$
\begin{aligned}
\mathrm{P}\left[\max _{s \leq T}-\hat{\mu} s+W_{s} \geq \hat{x}\right] & =1-\mathrm{P}\left[\max _{s \leq T}-\hat{\mu} s+W_{s} \leq \hat{x}\right] \\
& =1-\left(\mathcal{N}\left(\frac{\hat{x}+\hat{\mu} T}{\sqrt{T}}\right)-\mathrm{e}^{-2 \hat{\mu} \hat{x}} \mathcal{N}\left(\frac{-\hat{x}+\hat{\mu} T}{\sqrt{T}}\right)\right) \\
& =\mathcal{N}\left(\frac{-\hat{x}-\hat{\mu} T}{\sqrt{T}}\right)+\mathrm{e}^{-2 \hat{\mu} \hat{x}} \mathcal{N}\left(\frac{-\hat{x}+\hat{\mu} T}{\sqrt{T}}\right) .
\end{aligned}
$$

This completes the proof of (2.6).

We now turn to the proof of (2.7). Again by the independence of $\left\{W_{t} ; t \in \mathbb{R}_{+}\right\}$and $J_{1}$ and the symmetry of standard Brownian motion, we obtain

$$
\begin{aligned}
G_{B}(x, T) & =\psi(0) \mathrm{P}\left[\min _{s \leq J_{1}} x+\mu s+\sigma W_{s} \leq 0, J_{1} \leq T\right] \\
& =\psi(0) \int_{0}^{T} \mathrm{P}\left[\min _{s \leq t} x+\mu s+\sigma W_{s} \leq 0\right] \mathrm{d} F_{J_{1}}(t) \\
& =\psi(0) \int_{0}^{T} \mathrm{P}\left[\max _{s \leq t}-\hat{\mu} s+W_{s} \geq \hat{x}\right] \mathrm{d} F_{J_{1}}(t) .
\end{aligned}
$$

Replacing $T$ by $t$ in (2.10), we obtain (2.7).

Finally, from the independence of $\left\{W_{t} ; t \in \mathbb{R}_{+}\right\}, Y_{1}$, and $J_{1}$,

$$
\begin{aligned}
G_{C}(x, T)= & \int_{0}^{T} \mathrm{~d} F_{J_{1}}(t) \mathrm{E}\left[\psi\left(x+X_{t}^{c}-Y_{1}\right) \mathbf{1}\left(\min _{s \leq t} x+X_{s}^{c}>0, x+X_{t}^{c}-Y_{1}<0\right)\right] \\
= & \int_{0}^{T} \mathrm{~d} F_{J_{1}}(t) \\
& \times \int_{0}^{\infty} \mathrm{d} F(y) \mathrm{E}\left[\psi\left(x+X_{t}^{c}-y\right) \mathbf{1}\left(\min _{0 \leq s \leq t} x+X_{s}^{c}>0, x+X_{t}^{c}-y<0\right)\right],
\end{aligned}
$$

where in the last line we used the fact that $\mathrm{P}\left[\min _{s \leq t} x+X_{s}^{c}>0, x+X_{t}^{c}-y<0\right]=0$ for $y<0$. Also, observe that, using the symmetry of standard Brownian motion,

$$
\begin{aligned}
& \mathrm{E}\left[\psi\left(x+X_{t}^{c}-y\right) \mathbf{1}\left(\min _{0 \leq s \leq t} x+X_{s}^{c}>0, x+X_{t}^{c}-y<0\right)\right] \\
& \quad=\mathrm{E}\left[\psi\left(x-\sigma\left(-\hat{\mu} t+W_{t}\right)-y\right) \mathbf{1}\left(\max _{s \leq t}-\hat{\mu} s+W_{s} \leq \hat{x}, \hat{x}-\left(-\hat{\mu} t+W_{t}\right)-\hat{y} \leq 0\right)\right] .
\end{aligned}
$$

Now, applying the formula of the joint distribution of $W(\alpha ; t)$ and $M(\alpha ; t)$ with $\alpha=-\hat{\mu}$, we 
obtain, for all $t, y>0$,

$$
\begin{aligned}
\mathrm{E}[\psi( & \left.\left.x-\sigma\left(-\hat{\mu} t+W_{t}\right)-y\right) \mathbf{1}\left(\max _{s \leq t}-\hat{\mu} s+W_{s} \leq \hat{x}, \hat{x}-\left(-\hat{\mu} t+W_{t}\right)-\hat{y} \leq 0\right)\right] \\
= & \int \mathrm{d} w \int_{w^{+}}^{\infty} \mathrm{d} m \psi(x-\sigma w-y) \mathbf{1}(m \leq \hat{x}, \hat{x}-w-\hat{y} \leq 0) \\
& \times \frac{2(2 m-w)}{t \sqrt{2 \pi t}} \exp \left\{-\hat{\mu} w-\frac{1}{2} \hat{\mu}^{2} t-\frac{1}{2 t}(2 m-w)^{2}\right\} \\
= & \int_{\hat{x}-\hat{y}}^{\hat{x}} \mathrm{~d} w \psi(x-\sigma w-y) \exp \left\{-\hat{\mu} w-\frac{1}{2} \hat{\mu}^{2} t\right\} \\
& \times \int_{w^{+}}^{\hat{x}} \mathrm{~d} m \frac{2(2 m-w)}{t \sqrt{2 \pi t}} \exp \left\{-\frac{1}{2 t}(2 m-w)^{2}\right\} \\
= & \int_{\hat{x}-\hat{y}}^{\hat{x}} \mathrm{~d} w \psi(x-\sigma w-y) \exp \left\{-\hat{\mu} w-\frac{1}{2} \hat{\mu}^{2} t\right\} \\
& \times \int_{|w|}^{2 \hat{x}-w} \mathrm{~d} m \frac{m}{t \sqrt{2 \pi t}} \exp \left\{-\frac{1}{2 t} m^{2}\right\} \\
= & \int_{\hat{x}-\hat{y}}^{\hat{x}} \mathrm{~d} w \psi(x-\sigma w-y) \exp \left\{-\hat{\mu} w-\frac{1}{2} \hat{\mu}^{2} t\right\} \\
& \times \frac{1}{\sqrt{2 \pi t}}\left(\exp \left\{-\frac{w^{2}}{2 t}\right\}-\exp \left\{-\frac{(2 \hat{x}-w)^{2}}{2 t}\right\}\right) .
\end{aligned}
$$

Note that $(2 \hat{x}-w)^{2}+2 t \hat{\mu} w+\hat{\mu}^{2} t^{2}=(2 \hat{x}-\hat{\mu} t-w)^{2}+4 \hat{x} \hat{\mu} t$. Therefore,

$$
\begin{aligned}
& \int_{\hat{x}-\hat{y}}^{\hat{x}} \mathrm{~d} w \psi(x-\sigma w-y) \exp \left\{-\hat{\mu} w-\frac{1}{2} \hat{\mu}^{2} t\right\} \\
& \times \frac{1}{\sqrt{2 \pi t}}\left(\exp \left\{-\frac{w^{2}}{2 t}\right\}-\exp \left\{-\frac{(2 \hat{x}-w)^{2}}{2 t}\right\}\right) \\
& =\int_{\hat{x}-\hat{y}}^{\hat{x}} \mathrm{~d} w \psi(x-\sigma w-y) \\
& \quad \times \frac{1}{\sqrt{2 \pi t}}\left(\exp \left\{-\frac{(w+\hat{\mu} t)^{2}}{2 t}\right\}-\mathrm{e}^{-2 \hat{x} \hat{\mu}} \exp \left\{-\frac{(2 \hat{x}-\hat{\mu} t-w)^{2}}{2 t}\right\}\right) \\
& =\int_{0}^{y} \mathrm{~d} w \psi(w-y) \\
& \quad \times \frac{1}{\sigma \sqrt{2 \pi t}}\left(\exp \left\{-\frac{(x-w+\mu t)^{2}}{2 t \sigma^{2}}\right\}-\mathrm{e}^{-2 \hat{x} \hat{\mu}} \exp \left\{-\frac{(x+w-\mu t)^{2}}{2 t \sigma^{2}}\right\}\right) \\
& =\int_{0}^{y} \psi(w-y)\left[g\left(x-w+\mu t ; t \sigma^{2}\right)-\mathrm{e}^{-2 \hat{x} \hat{\mu}} g\left(x+w-\mu t ; t \sigma^{2}\right)\right] \mathrm{d} w .
\end{aligned}
$$

This gives (2.8).

To calculate $G_{D}$, we use the strong Markov property of the process $X$.

Proposition 2.2. We have

$$
G_{D}(x, T)=\mathrm{E}_{x}\left[\mathbf{1}_{\tilde{D}} \Phi\left(X_{J_{1}}, T-J_{1}\right)\right]=L \Phi(x, T) .
$$


Here, $\tilde{D}=\left[J_{1} \leq T, \inf _{s \leq J_{1}} X_{S}>0\right]$, the integral operator $L$ is defined by

$$
L f(x, t)=\int_{0}^{t} \mathrm{~d} F_{J_{1}}(s) \int \mathrm{d} F(y) \int_{y^{+}}^{\infty} \mathrm{d} w f(w-y, t-s) H(x, w, s)
$$

for any nonnegative Borel measurable function $f$ on $\mathbb{R}_{+}^{2}$, and $H$ is defined in (2.9).

Proof. Note that $\tilde{D} \in \mathcal{F}_{J_{1}}$. Therefore, by the strong Markov property we have

$$
G_{D}(x, T)=\mathrm{E}_{x}\left[\mathrm{E}_{x}\left[\mathbf{1}_{\tilde{D}} \psi\left(X_{\tau(T)}\right) \mathbf{1}(\tau(T) \leq T) \mid \mathcal{F}_{J_{1}}\right]\right]=\mathrm{E}_{x}\left[\mathbf{1}_{\tilde{D}} \Phi\left(X_{J_{1}}, T-J_{1}\right)\right] .
$$

Recall that $Y_{1}=-\log \left(1+U_{1}\right)$. Therefore, by the independence of $J_{1}, Y_{1}$, and $\left\{W_{t} ; t \geq 0\right\}$, we obtain

$$
\begin{aligned}
G_{D}(x, T)=\mathrm{E}\left[\mathbf{1}\left(J_{1} \leq T, \min _{s \leq J_{1}} x+X_{s}^{c}>0, x+X_{J_{1}}^{c}-Y_{1}>0\right)\right. \\
\left.\times \Phi\left(x+X_{J_{1}}^{c}-Y_{1}, T-J_{1}\right)\right] \\
=\int_{0}^{T} \mathrm{~d} F_{J_{1}}(t) \int \mathrm{d} F(y) \mathrm{E}\left[\mathbf{1}\left(\min _{s \leq t} x+X_{s}^{c}>0, x+X_{t}^{c}-y>0\right)\right. \\
\left.\times \Phi\left(x+X_{t}^{c}-y, T-t\right)\right] .
\end{aligned}
$$

Let us calculate the integrand. Using the symmetry of Brownian motions, we obtain

$$
\begin{aligned}
& \mathrm{E}\left[\mathbf{1}\left(\min _{s \leq t} x+X_{s}^{c}>0, x+X_{t}^{c}-y>0\right) \Phi\left(x+X_{t}^{c}-y, T-t\right)\right] \\
&=\mathrm{E} {\left[\mathbf{1}\left(\max _{s \leq t}-x-\mu s+\sigma W_{s}<0,-x-\mu t+\sigma W_{t}+y<0\right)\right.} \\
&\left.\times \Phi\left(x+\mu t-\sigma W_{t}-y, T-t\right)\right] \\
&=\mathrm{E} {\left[\mathbf{1}\left(\max _{s \leq t}-\hat{x}-\hat{\mu} s+W_{s}<0,-\hat{x}-\hat{\mu} t+W_{t}+y<0\right)\right.} \\
&\left.\times \Phi\left(x-\sigma\left(-\hat{\mu} t+W_{t}\right)-y, T-t\right)\right] .
\end{aligned}
$$

Using Theorem 2.1 with $\alpha=-\hat{\mu}$, we obtain

$$
\begin{aligned}
& \mathrm{E}\left[\mathbf{1}\left(\max _{s \leq t}-\hat{x}-\hat{\mu} s+W_{s}<0,-\hat{x}-\hat{\mu} t+W_{t}+y<0\right)\right. \\
& \left.\times \Phi\left(x-\sigma\left(-\hat{\mu} t+W_{t}\right)-y, T-t\right)\right] \\
& =\int_{-\infty}^{(\hat{x}-\hat{y}) \wedge \hat{x}} \mathrm{~d} w \int_{w^{+}}^{\hat{x}} \mathrm{~d} v \Phi(x-\sigma w-y, T-t) \\
& \quad \times \frac{2(2 v-w)}{t \sqrt{2 \pi t}} \exp \left\{-\hat{\mu} w-\frac{1}{2} \hat{\mu}^{2} t-\frac{1}{2 t}(2 v-z)^{2}\right\} \\
& =\int_{-\infty}^{(\hat{x}-\hat{y}) \wedge \hat{x}} \mathrm{~d} w \Phi(x-\sigma w-y, T-t) \exp \left\{-\hat{\mu} w-\frac{1}{2} \hat{\mu}^{2} t\right\} \\
& \quad \times \int_{w^{+}}^{\hat{x}} \frac{2(2 v-w)}{t \sqrt{2 \pi t}} \exp \left\{-\frac{(2 v-z)^{2}}{2 t}\right\} \mathrm{d} v .
\end{aligned}
$$


Similar to the calculation of $G_{C}$, we have

$$
\begin{aligned}
& \int_{-\infty}^{(\hat{x}-\hat{y}) \wedge \hat{x}} \mathrm{~d} w \Phi(x-\sigma w-y, T-t) \exp \left\{-\hat{\mu} w-\frac{1}{2} \hat{\mu}^{2} t\right\} \\
& \times \int_{w^{+}}^{\hat{x}} \frac{2(2 v-w)}{t \sqrt{2 \pi t}} \exp \left\{-\frac{(2 v-z)^{2}}{2 t}\right\} \mathrm{d} v \\
& \quad=\int_{-\infty}^{(\hat{x}-\hat{y}) \wedge \hat{x}} \Phi(x-\sigma w-y, T-t)\left[g(w+\hat{\mu} t ; t)-g(w+t \hat{\mu}-2 \hat{x} ; t) \mathrm{e}^{-2 \hat{\mu} \hat{x}}\right] \mathrm{d} w \\
& \quad=\int_{y^{+}}^{\infty} \Phi(w-y, T-t)\left[g\left(w-x-\mu t ; t \sigma^{2}\right)-g\left(w+x-\mu t ; t \sigma^{2}\right) \mathrm{e}^{-2 \hat{\mu} \hat{x}}\right] \mathrm{d} w,
\end{aligned}
$$

where we used the change of variable $x-\sigma w \mapsto w$ in the last equation. This completes the proof.

Theorem 2.2. For every bounded measurable $\psi$, the function $\Phi$ defined in (1.6) satisfies the integral equation

$$
\Phi(x, T)=G(x, T)+L \Phi(x, T) .
$$

Here, the integral operator $L$ is defined by (2.12), $G=G_{A}+G_{B}+G_{C}$, and $G_{A}, G_{B}$, and $G_{C}$ are given as in (2.6), (2.7), and (2.8), respectively.

Remark. The decomposition of $\Phi$ into $G_{A}, G_{B}, G_{C}$, and $G_{D}$ is actually quite intuitive. Assume that the interest rate is 0 . Consider a financial security with time to maturity $T$ which pays $\psi\left(X_{\tau(T)}\right)$ at time $\tau(T) \leq T$. Assume that $\mathrm{E}_{x}$ is the 'right' measure under which we can calculate prices. Then the time- 0 price of such security is $\Phi$. We now decompose this security into four securities by classifying the possible causes of the payment $\psi\left(X_{\tau(T)}\right)$.

We track the sample path of $X$ up to time $T$ and see whether a jump has occurred. If there is no jump, namely, $T \leq J_{1}$, then the cause of the payment must be diffusion. Namely, $X_{\tau(T)}=0$. The time- 0 price of this type of payment is given by $G_{A}$. Suppose the contrary, that is, $J_{1} \leq T$. We further classify the possible causes of the payment. If $\tau(T)<J_{1}$ then the cause must be diffusion again. The time- 0 price of this type of payment is given by $G_{B}$. If $\tau(T)=J_{1}$ then the cause of the payment is a jump and the time- 0 price of this type of payment is $G_{C}$. Now, the remaining possibility is $J_{1}<\tau(T) \leq T$. At time $J_{1}$, from the renewal property of $X$, the security can be seen as a 'new' security almost the same as the old one except that the time to maturity is $T-J_{1}$. The time- 0 price of this 'new' security is $G_{D}$.

In Appendix A we will further extend the above classification of causes of the payment $\psi\left(X_{\tau(T)}\right)$ to obtain an infinite-series expansion of the bond price.

\section{Analytic properties of bond prices}

To fix ideas, we adopt, from [6, Section 2.2.1] and [9], the following definition of yield spreads and credit spreads.

Definition 3.1. For the bond price defined in (1.5), the promised yield for maturity $T$ is given by $y\left(V_{0}, T\right)=(1 / T) \log \left(1 / D\left(V_{0}, T\right)\right)$ and the credit spread for maturity $T$ is given by $s\left(V_{0}, T\right)=$ $y\left(V_{0}, T\right)-r$.

Note that, in Definition 3.1, 1 is the face value of the bond, and it follows immediately from the definition that $D\left(V_{0}, T\right) \exp \left\{y\left(V_{0}, T\right) T\right\}=1$. 
Lemma 3.1. For all $x>0$ and $y>0$, the function

$$
t \mapsto \mathrm{E}\left[\psi\left(x+X_{t}^{c}-y\right) \mathbf{1}\left(\min _{s \leq t} x+X_{s}^{c}>0, x+X_{t}^{c}-y<0\right)\right]
$$

is continuous on $(0, \infty)$.

Proof. Recall that in the proof of Proposition 2.1 we have

$$
\begin{gathered}
\mathrm{E}\left[\psi\left(x+X_{t}^{c}-y\right) \mathbf{1}\left(\min _{s \leq t} x+X_{s}^{c}>0, x+X_{t}^{c}-y<0\right)\right] \\
=\int_{0}^{y} \psi(w-y) H(x, w, t) \mathrm{d} w
\end{gathered}
$$

where $H$ is given by (2.9). From this, it follows easily that the function in (3.1) is continuous. This completes the proof.

Lemma 3.2. Assume that $x>0$. Then

$$
\lim _{T \rightarrow 0+} \frac{\partial}{\partial T} \mathrm{P}\left[\min _{s \leq T} x+\mu s+\sigma W_{s}<0\right]=0 .
$$

Also, for all $n \in \mathbb{N}$, we have

$$
\mathrm{P}\left[\min _{t \leq T} x+\mu t+\sigma W_{s}<0\right]=o\left(T^{n}\right) \quad \text { as } T \rightarrow 0+.
$$

Proof. Firstly, we prove (3.2). By the symmetry of standard Brownian motion we have

$$
\mathrm{P}\left[\min _{s \leq T} x+\mu s+\sigma W_{s} \leq 0\right]=\mathrm{P}\left[\max _{s \leq T}-\mu s+\sigma W_{s} \geq x\right]=\mathrm{P}\left[\max _{s \leq T}-\hat{\mu} s+W_{s} \geq \hat{x}\right] .
$$

Note that $x>0$. Therefore, by (2.5) we have

$$
\mathrm{P}\left[\max _{s \leq T}-\hat{\mu} s+W_{s} \geq \hat{x}\right]=\mathcal{N}\left(-\frac{\hat{x}+\hat{\mu} T}{\sqrt{T}}\right)+\mathrm{e}^{-2 \hat{\mu} \hat{x}} \mathcal{N}\left(\frac{-\hat{x}+\hat{\mu} T}{\sqrt{T}}\right),
$$

which converges to 0 as $T \rightarrow 0^{+}$. Recall that $g\left(x ; \sigma^{2}\right)=1 /\left(\sqrt{2 \pi \sigma^{2}}\right) \exp \left\{-x^{2} / 2 \sigma^{2}\right\}$. We have

$$
\begin{aligned}
& \frac{\partial}{\partial T}\left(\mathcal{N}\left(-\frac{\hat{x}+\hat{\mu} T}{\sqrt{T}}\right)+\mathrm{e}^{-2 \hat{\mu} \hat{x}} \mathcal{N}\left(\frac{-\hat{x}+\hat{\mu} T}{\sqrt{T}}\right)\right) \\
& =-g\left(\frac{-(\hat{x}+\hat{\mu} T)}{\sqrt{T}} ; 1\right) \frac{\sqrt{T} \hat{\mu}-(\hat{x}+\hat{\mu} T) T^{-1 / 2} / 2}{T} \\
& +\mathrm{e}^{-2 \hat{\mu} \hat{x}} g\left(\frac{-\hat{x}+\hat{\mu} T}{\sqrt{T}} ; 1\right) \frac{\sqrt{T} \hat{\mu}-(-\hat{x}+\hat{\mu} T) T^{-1 / 2} / 2}{T} \\
& =\frac{1}{2}\left(-g\left(\frac{-(\hat{x}+\hat{\mu} T)}{\sqrt{T}} ; 1\right) \frac{\hat{\mu} T-\hat{x}}{T^{3 / 2}}+g\left(\frac{\hat{x}+\hat{\mu} T}{\sqrt{T}} ; 1\right) \frac{\hat{\mu} T+\hat{x}}{T^{3 / 2}}\right) \\
& =g\left(\frac{\hat{x}+\hat{\mu} T}{\sqrt{T}} ; 1\right) \frac{\hat{x}}{T^{3 / 2}} \\
& =\frac{1}{\sqrt{2 \pi}} \hat{x} \exp \left\{-\frac{1}{2}\left(\frac{\hat{x}}{\sqrt{T}}+\hat{\mu} \sqrt{T}\right)^{2}\right\} \frac{1}{T^{3 / 2}} \text {. }
\end{aligned}
$$


Observe that, for all $T>0$,

$$
0 \leq \frac{1}{\sqrt{2 \pi}} \hat{x} \exp \left\{-\frac{1}{2}\left(\frac{\hat{x}}{\sqrt{T}}+\hat{\mu} \sqrt{T}\right)^{2}\right\} \frac{1}{T^{3 / 2}} \leq C \exp \left\{-\frac{\hat{x}^{2}}{2 T}\right\} \frac{1}{T^{3 / 2}}
$$

for some constant $C>0$ independent of $T>0$. This implies that (3.2) holds.

We prove (3.3). Let $n \in \mathbb{N}$. Then, by l'Hôpital's rule,

$$
\begin{aligned}
\lim _{T \rightarrow 0+} & \frac{\mathrm{P}\left[\min _{s \leq T} x+\mu s+\sigma W_{s}<0\right]}{T^{n}} \\
& \leq \lim _{T \rightarrow 0+} \frac{(\partial / \partial T) \mathrm{P}\left[\min _{s \leq T} x+\mu s+\sigma W_{s}<0\right]}{n T^{n-1}} \\
& \leq \lim _{T \rightarrow 0+} C \exp \left\{-\frac{\hat{x}^{2}}{2 T}\right\} \frac{1}{n T^{(n-1)+3 / 2}} \\
& =0 .
\end{aligned}
$$

This completes the proof.

Recall that $D\left(V_{0}, T\right)$ is the bond price defined in (1.5) and that we set $x=\log \left(V_{0} / K_{0}\right)$.

Proposition 3.1. We have the following analytic properties of bond prices.

(a) For each $T>0, \lim _{V_{0} \rightarrow \infty} D\left(V_{0}, T\right)=\mathrm{e}^{-r T}$.

(b) For each $V_{0} \in\left(K_{0}, \infty\right), \lim _{T \rightarrow 0+} \mathrm{P}_{x}[\tau(T) \leq T]=0$.

Proof. We prove (a) first. Since the function $\psi$ is bounded, by (1.5) and (1.6), it suffices to show that $\lim _{x \rightarrow \infty} \mathrm{P}_{x}[\tau(T) \leq T]=0$. Now since $X=\left(X_{t}\right)$ is càdlàg (right continuous with left limits), it is clear that, for fixed $T, \tau(T, x)=\inf \left\{0 \leq t \leq T ; x+X_{t}^{c}-Z_{t} \leq 0\right\} \rightarrow \infty$ as $x \uparrow \infty$. This implies that $\lim _{x \rightarrow \infty} \mathrm{P}_{x}[\tau(T) \leq T]=0$.

Next, consider (b). Write

$$
\mathrm{P}_{x}[\tau(T) \leq T]=\mathrm{P}_{x}[A]+\mathrm{P}_{x}[B \cup C \cup D],
$$

where $\{A, B, C, D\}$ is the partition of $[\tau(T) \leq T]$ in (2.2). For the second term on the righthand side of (3.4), we have

$$
\mathrm{P}_{x}[B \cup C \cup D]=\mathrm{P}_{x}\left[\tau(T) \leq T, J_{1} \leq T\right] \leq \mathrm{P}\left[J_{1} \leq T\right]=1-\mathrm{e}^{-\lambda T} \rightarrow 0, \quad T \rightarrow 0+.
$$

On the other hand, note that

$$
\mathrm{P}_{x}[A]=\mathrm{P}_{x}\left[\tau(T) \leq T<J_{1}\right]=\mathrm{P}\left[T<J_{1}\right] \mathrm{P}\left[\min _{s \leq T} x+c s+\sigma W_{s} \leq 0\right] .
$$

By (3.3) we obtain

$$
\lim _{T \rightarrow 0+} \mathrm{P}_{x}[A] \leq \lim _{T \rightarrow 0+} \mathrm{P}\left[\min _{s \leq T} x+c s+\sigma W_{s} \leq 0\right]=0 .
$$

Combining these results with (3.4), we have (b). The proof is then complete. 
Theorem 3.1. Assume that $\psi$ is continuous and that $0 \leq \psi \leq 1$. Then, for each $V_{0} \in\left(K_{0}, \infty\right)$,

$$
\lambda \int_{y>x} \psi(x-y) \mathrm{d} F(y) \leq \liminf _{T \rightarrow 0+} s\left(V_{0}, T\right) \leq \limsup _{T \rightarrow 0+} s\left(V_{0}, T\right) \leq \lambda \int_{y \geq x} \psi(x-y) \mathrm{d} F(y) .
$$

In particular,

(a) if $\psi>0$ and $\mathrm{P}\left[Y_{1}>x\right]>0$, we have a strictly positive credit spread for zero maturity;

(b) if $\psi(0)=0$ or $F$ is continuous at $x$, then we have the following asymptotic behavior of the credit spread:

$$
\lim _{T \rightarrow 0+} s\left(V_{0}, T\right)=\lambda \int_{y>x} \psi(x-y) \mathrm{d} F(y) .
$$

Proof. First, we prove the lower bound in (3.5). Since $0 \leq \psi \leq 1$, we have $1 \geq \Phi(x, T) \geq$ $G_{C}(x, T)$. Note that $\Phi(x, T) \rightarrow 0$ as $T \rightarrow 0+$ by Proposition 3.1(b). Therefore,

$$
\begin{aligned}
\liminf _{T \rightarrow 0+} s\left(V_{0}, T\right) & =\liminf _{T \rightarrow 0+}\left[\frac{1}{T} \log \left(\frac{\mathrm{e}^{r T}}{1-\Phi(x, T)}\right)-r\right] \\
& =\liminf _{T \rightarrow 0+} \frac{1}{T} \log \left(\frac{1}{1-\Phi(x, T)}\right) \\
& \geq \liminf _{T \rightarrow 0+} \frac{1}{T} \log \left(\frac{1}{1-G_{C}(x, T)}\right) .
\end{aligned}
$$

Note that, by Proposition 3.1(b), $\Phi(x, T) \rightarrow 0$ as $T \rightarrow 0+$. Hence, we obtain $0 \leq G_{C}(x, T) \leq$ $\Phi(x, T) \rightarrow 0$ as $T \rightarrow 0+$. By the mean value theorem we obtain

$$
\liminf _{T \rightarrow 0+} \frac{1}{T} \log \left(\frac{1}{1-G_{C}(x, T)}\right) \geq \liminf _{T \rightarrow 0+} \frac{\partial G_{C}(x, T) / \partial T}{1-G_{C}(x, T)}=\liminf _{T \rightarrow 0+} \frac{\partial G_{C}}{\partial T}(x, T) .
$$

By (2.8), Lemma 3.1, and the fundamental theorem of calculus, we obtain

$$
\begin{aligned}
\frac{\partial G_{C}}{\partial T}(x, T)= & \lambda \mathrm{e}^{-\lambda T} \\
& \times \int_{0}^{\infty} \mathrm{d} F(y) \mathrm{E}\left[\psi\left(x+X_{T}^{c}-y\right) \mathbf{1}\left(\min _{s \leq T} x+X_{s}^{c}>0, x+X_{T}^{c}-y<0\right)\right] .
\end{aligned}
$$

Since $\psi$ is continuous and $0 \leq \psi \leq 1$, we obtain

$$
\liminf _{T \rightarrow 0+} s\left(V_{0}, T\right) \geq \liminf _{T \rightarrow 0+} \frac{\partial G_{C}}{\partial T}(x, T) \geq \lambda \int_{y>x} \psi(x-y) \mathrm{d} F(y) .
$$

This proves the lower bound in (3.5).

Next, we show the upper bound in (3.5). By the definition of the function $G$, there exists a function $I$ of $t$ such that $t \mapsto I(t)$ is continuous on $(0,1], \lim _{t \rightarrow 0+} I(t)=0$, and

$$
\sup _{y \in[x / 2,3 x / 2]} G(y, t) \leq I(t) .
$$


(See also (2.6), (2.7), and (2.11).) By Proposition 2.2,

$$
L G(x, T) \leq \int_{0}^{T} I(t) \lambda \mathrm{e}^{-\lambda(T-t)} \mathrm{d} t+\int_{0}^{T} \mathrm{P}_{x}\left[\inf _{0 \leq s \leq t} X_{s} \in(0, \infty) \backslash\left[\frac{x}{2}, \frac{3 x}{2}\right]\right] \lambda \mathrm{e}^{-\lambda t} \mathrm{~d} t .
$$

By Lemma A.1,

$$
L^{2} \Phi(x, T) \leq 1-\mathrm{e}^{-\lambda T}-\lambda T \mathrm{e}^{-\lambda T}
$$

An iteration of (2.13) gives

$$
\begin{aligned}
\Phi(x, T)= & G(x, T)+L G(x, T)+L^{2} \Phi(x, T) \\
\leq & G(x, T)+\int_{0}^{T} I(t) \lambda \mathrm{e}^{-\lambda(T-t)} \mathrm{d} t \\
& +\int_{0}^{T} \mathrm{P}_{x}\left[\inf _{0 \leq s \leq t} X_{s} \in(0, \infty) \backslash\left[\frac{x}{2}, \frac{3 x}{2}\right]\right] \lambda \mathrm{e}^{-\lambda t} \mathrm{~d} t+1-\mathrm{e}^{-\lambda T}-\lambda T \mathrm{e}^{-\lambda T},
\end{aligned}
$$

where the last inequality follows from (3.8) and (3.9). Observe that among the terms in (3.10),

$$
\frac{\partial}{\partial T} \int_{0}^{T} I(t) \lambda \mathrm{e}^{-\lambda(T-t)} \mathrm{d} t=I(T) \lambda-\lambda \int_{0}^{T} I(t) \lambda \mathrm{e}^{-\lambda(T-t)} \mathrm{d} t \rightarrow 0, \quad T \rightarrow 0+,
$$

and

$$
\begin{aligned}
& \frac{\partial}{\partial T} \int_{0}^{T} \mathrm{P}_{x}\left[\inf _{0 \leq s \leq t} X_{s} \in(0, \infty) \backslash\left[\frac{x}{2}, \frac{3 x}{2}\right]\right] \lambda \mathrm{e}^{-\lambda t} \mathrm{~d} t \\
& \quad=\mathrm{P}_{x}\left[\inf _{0 \leq s \leq T} X_{s} \in(0, \infty) \backslash\left[\frac{x}{2}, \frac{3 x}{2}\right]\right] \lambda \mathrm{e}^{-\lambda T} \rightarrow 0, \quad T \rightarrow 0+.
\end{aligned}
$$

In (3.11) we used the fact that $t \mapsto \inf _{0 \leq s \leq t} X_{S}$ is almost surely continuous at each $T>0$ by the quasi-left continuity of $X$. In addition, as $T \rightarrow 0, \partial G_{A}(x, T) / \partial T \rightarrow 0$ by Lemma 3.2 and $\partial G_{B}(x, T) / \partial T \rightarrow 0$. With an argument similar to (3.6), we deduce, from (3.10) and the above estimates for the derivatives, that

$$
\limsup _{T \rightarrow 0+} s\left(V_{0}, T\right) \leq \limsup _{T \rightarrow 0+} \frac{\partial G_{C}}{\partial T}(x, T) \leq \lambda \int_{y \geq x} \psi(x-y) \mathrm{d} F(y),
$$

where the last equality follows from (3.7). We have obtained the upper bound in (3.5). This completes the proof.

Remark. It is worth noting that Hilberink and Rogers [5] proved a similar result for the credit spread in the Leland model. Instead of considering the case where $X$ has two-sided jumps, only downward jumps were allowed in the definition of $X$.

\section{Appendix A. An infinite-series expansion for the bond price}

Recall the definition of the operator $L$ in Proposition 2.2. Theorem 2.2 states that we can write $\Phi$ as

$$
\Phi(x, t)=G(x, t)+L \Phi(x, t) \text { for all }(x, t) \in \mathbb{R}_{+}^{2} .
$$


After $n$-fold iteration of (A.1), we have

$$
\Phi(x, t)=\sum_{k=0}^{n} L^{k} G(x, t)+L^{n+1} \Phi(x, t),
$$

where $L^{0} f=f$ and $L^{k+1} f=L\left(L^{k} f\right)$. On the other hand, by the definition of $L$,

$$
L f(x, t)=\mathrm{E}_{x}\left[f\left(X_{J_{1}}, t-J_{1}\right) \mathbf{1}\left(J_{1} \leq t, \inf _{0 \leq s \leq J_{1}} X_{s}>0\right)\right] .
$$

(See Proposition 2.2.) The probabilistic interpretation of $L^{n} f, n>2$, is given by the following lemma.

Lemma A.1. Let $f$ be a nonnegative measurable function defined on $\mathbb{R}_{+}^{2}$. Then, for any $n \in \mathbb{N}$,

$$
L^{n} f(x, t)=\mathrm{E}_{x}\left[f\left(X_{J_{n}}, t-J_{n}\right) \mathbf{1}\left(J_{n} \leq t, \inf _{0 \leq s \leq J_{n}} X_{s}>0\right)\right] .
$$

Moreover, for any $T \in(0, \infty)$ and any $n \geq 1$,

$$
\sup _{(x, t) \in \mathbb{R}_{+} \times[0, T]} L^{n} f(x, t) \leq\|f\|_{\infty} \sum_{m \geq n} \mathrm{e}^{-\lambda T} \frac{(\lambda T)^{m}}{m !} .
$$

Proof. The proof proceeds by induction. We already have the case in which $n=1$ by the definition of $L$. Assume that, for $n=k$, the conclusion of the lemma holds. Then, for $n=k+1$,

$$
\begin{aligned}
L^{k+1} f(x, t)= & \mathrm{E}_{x}\left[L^{k} f\left(X_{J_{1}}, t-J_{1}\right) \mathbf{1}\left(J_{1} \leq t, \inf _{0 \leq s \leq J_{1}} X_{s}>0\right)\right] \\
=\mathrm{E}_{x}[ & \left.\mathrm{E}_{X_{J_{1}}}\left[f\left(X_{J_{k}}, v-J_{k}\right) \mathbf{1}\left(J_{k} \leq v, \inf _{0 \leq s \leq J_{k}} X_{s}>0\right)\right]\right|_{v=t-J_{1}} \\
& \left.\times \mathbf{1}\left(J_{1} \leq t, \inf _{0 \leq s \leq J_{1}} X_{s}>0\right)\right],
\end{aligned}
$$

where we have applied the case of (A.3) for $n=k$ in the last line. On the other hand, by the strong Markov property of $X$ we have

$$
\begin{aligned}
\mathrm{E}_{x}\left[f\left(X_{J_{k+1}}, t-J_{k+1}\right) \mathbf{1}\left(J_{k+1} \leq t, \inf _{0 \leq s \leq J_{k+1}} X_{s}>0\right)\right] \\
=\mathrm{E}_{x}\left[\mathrm{E}_{x}\left[f\left(X_{J_{k+1}}, t-J_{k+1}\right) \mathbf{1}\left(J_{k+1} \leq t, \inf _{J_{1} \leq s \leq J_{k+1}} X_{s}>0\right) \mid \mathcal{F}_{J_{1}}\right]\right. \\
\left.\quad \times \mathbf{1}\left(J_{1} \leq t, \inf _{0 \leq s \leq J_{1}} X_{s}>0\right)\right] \\
=\mathrm{E}_{x}\left[\left.\mathrm{E}_{X_{J_{1}}}\left[f\left(X_{J_{k}}, v-J_{k}\right) \mathbf{1}\left(J_{k} \leq v, \inf _{0 \leq s \leq J_{k}} X_{s}>0\right)\right]\right|_{v=t-J_{1}}\right. \\
\left.\quad \times \mathbf{1}\left(J_{1} \leq t, \inf _{0 \leq s \leq J_{1}} X_{s}>0\right)\right] .
\end{aligned}
$$

Hence, we have proved that (A.3) holds for $n=k+1$. By the induction hypothesis we have proved the first part of the lemma.

The second part of the lemma now follows immediately from the nonnegativity of $f$ and the fact that $J_{n}$ is a sum of $n$ independent exponential random variables with mean $1 / \lambda$. The proof is now complete. 
From this, we obtain from (A.2) that, for every bounded $\psi$,

$$
\Phi(x, T)=\lim _{n \rightarrow \infty}\left(\sum_{k=0}^{n} L^{k} G(x, T)+L^{n+1} \Phi(x, T)\right)=\sum_{k=0}^{\infty} L^{k} G(x, T) .
$$

Write $x=\log \left(V_{0} / K_{0}\right)$. By (1.5) we obtain the following infinite-series expansion for the bond price.

Theorem A.1. For any bounded function $\psi$, the bond price $D\left(V_{0}, T\right)$ in (1.5) has the following expansion:

$$
D\left(V_{0}, T\right)=\mathrm{e}^{-r T}-\mathrm{e}^{-r T} \sum_{m=0}^{\infty} L^{m} G\left(\log \left(\frac{V_{0}}{K_{0}}\right), T\right),
$$

where $G=G_{A}+G_{B}+G_{C}$, and $G_{A}, G_{B}$, and $G_{C}$ are given as in (2.6), (2.7), and (2.8), respectively. Moreover, the series converges uniformly in $\left(\log \left(V_{0} / K_{0}\right), T\right) \in \mathbb{R}_{+} \times\left[0, T^{*}\right]$ for any $T^{*} \in(0, \infty)$.

\section{References}

[1] Altman, E. I. and Bencivenga, J. C. (1995). A yield premium model for the high-yield debt market. Finanicial Analyst J. 51, 49-56.

[2] Bingham, N. H. And Kiesel, R. (2004). Risk-Neutral Valuation, 2nd edn. Springer, London.

[3] Black, F. AND CoX, J. C. (1976). Valuing corporate securities: some effects of bond indenture provisions. $J$. Finance 31, 351-367.

[4] Franks, J. R. AND Torous, W. N. (1994). A comparison of financial recontracting in distressed exchanges and Chapter 11 organizations. J. Financial Econom. 35, 349-370.

[5] Hilberink, B. and Rogers, L. C. G. (2002). Optimal capital structure and endogenous default. Finance Stoch. 6, 237-363.

[6] Lando, D. (2004). Credit Risk Modeling. Princeton University Press.

[7] Leland, H. E. (1994). Corporate debt value, bond covenants, and optimal capital structure. J. Finance 49, $1213-1252$.

[8] LongstafF, F. A. And Schwartz, E. S. (1995). A simple approach to valuing risky fixed and floating rate debt. J. Finance 50, 789-819.

[9] Merton, R. C. (1974). On the pricing of corporate debt: the risk structure of interest rates. J. Finance 29, 449-470.

[10] Shreve, S. E. (2004). Stochastic Calculus for Finance. II. Springer, New York.

[11] ZHOU, C. (2001). The term structure of credit spreads with jump risk. J. Banking Finance 26, 2015-2040. 